\title{
ON BASIC CONDITIONS FOR LONG-WAVE SIMULATIONS IN HARBORS BY THE BOUSSINESQ MODEL
}

\author{
Kazuyuki Ota ${ }^{1}$, Akinori Yoshida ${ }^{2}$, Masaru Yamashiro ${ }^{3}$ and Yasuhiro Nishii ${ }^{4}$
}

\begin{abstract}
For wave field calculations to estimate long-period ship motions in harbors, the so-called Boussinesq model seems to be widely used because of its versatility. However, it has not yet been clarified how large the sponge layer should be to simulate an open-sea condition, how long the computation should be continued to obtain a statistically stable spectrum, and what size of computational area should be considered. In this study, these basic conditions were investigated by conducting wave field simulations using the Boussinesq model. Useful information was obtained such as: (1) the thickness of the sponge layer can be much less than the thickness expected from one-dimensional calculations, (2) the data length to obtain a stable spectrum needs to be much longer than statistically expected 3 to 5 hours, and this is because the beat waves are produced by selectively amplified component waves near resonant frequencies in harbors.(3) the size of the computational area does not affect the results greatly and the distance $3 \mathrm{~km}$ between the end of the breakwater and the lateral sponge boundary is practically sufficient for the harbor mouth opening.
\end{abstract}

Keywords: long-period ship motions; long waves; Boussinesq model; harbor oscillations

\section{INTRODUCTION}

To estimate long period motions of moored ships in harbors, two techniques for numerical simulations are generally needed: one for the calculation of the ship motion and the other the calculation of the wave field in the harbor. As for the motion of the ships, the retardation function method (e.g. Wehausen 1971), which can take the decaying effects of generated waves by ship motions into account, seems to be the best to deal with non-linear motions under non-linear mooring forces. As for wave field calculations, the Boussinesq model (e.g. Madsen et al. 1991) seems to be widely accepted because of its versatile features.

Wave exciting forces exerted on the ship are needed to be evaluated from the wave field around the ship to solve the equation of motions by the retardation function method. Bingham (2000) presented a method to evaluate the wave exciting forces from the wave field calculations by the Boussinesq model using panel-method, in which the velocity potentials on the ship hull and the water surface in the vicinity of the ship are expressed by a number of panels of constant potential-strength. Yoshida et al. (2008) presented a method to evaluate the wave exciting forces by using eigen function expansions and Green's Identity Formula for the expression of the velocity potentials of the fluid motion around the ship.

Since ship motions are sensitive to waves near the natural frequencies of motions, it is crucial to estimate accurately the long-wave spectra in evaluating wave exciting forces exerted on ships at berth. A number of estimations on long waves by the Boussinesq model have been reported recently (e.g., Fujihata et al., 1998; Hiraishi and Hirayama, 2003). However, little is known about how large the sponge layer should be taken to simulate open-sea conditions; how long the computation should be continued to obtain statistically stable spectra; and what the size of the computational area should be considered. In this study these basic conditions were investigated by conducting wave field simulations by the Boussinesq model.

\section{NUMERICAL MODEL}

The Boussinesq model NOWT-PARI Ver.4.6 $\beta$ used in this research was originally provided by Hirayama (2002) and has been partially modified by one of the authors. The computational area (Figure 1) is around the Shibushi harbor in Kagoshima prefecture, Japan. The wave generating boundary is set about $6.2 \mathrm{~km}$ from the land boundary. The longshore length is set $9 \mathrm{~km}$ unless the size of the computational area is being investigated. To make it simple to clarify the association between the computational conditions and results, the contour lines of the sea bottom topography and the coastline are modified to be straight lines. The coastline, breakwater, and quay wall are set to be

\footnotetext{
${ }^{1}$ Civil Engineering Research Laboratory, Central Research Institute of Electric Power Industry, 1646 Abiko, Abiko-city, Chiba, 270-1194, Japan

${ }^{2}$ Sanyo Consultant Co., Ltd., Kyomachi 3-14-7, Kokurakita, Kitakyushu, 802-8534,Japan

${ }^{3}$ Dept. of Civil Eng., Kyushu Univ., 744, Motooka, Nishi-ku, Fukuoka-city, Fukuoka, 819-0395, Japan

${ }^{4}$ Dept. of Civil Eng., Kyushu Univ., 744, Motooka, Nishi-ku, Fukuoka-city, Fukuoka, 819-0395, Japan
} 


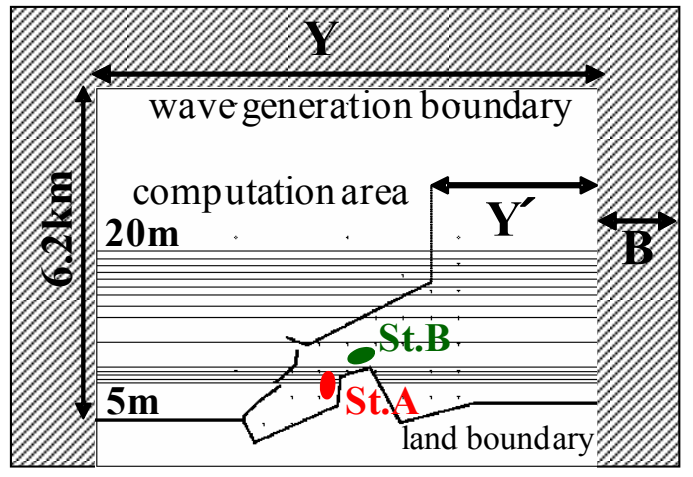

Figure 1. Computational area around Shibushi harbor.

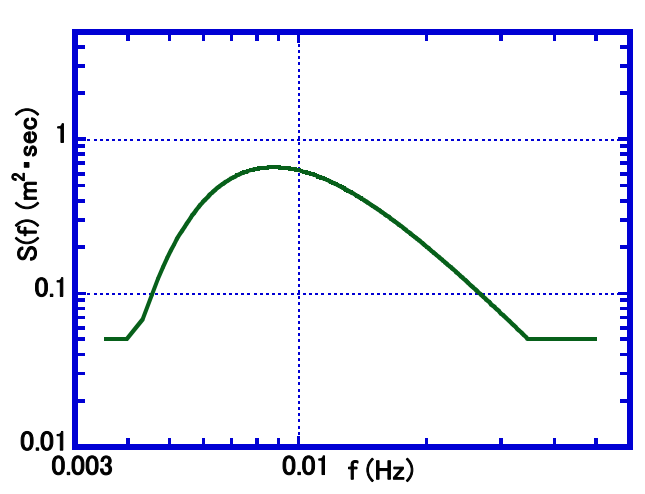

Figure 2. Incident long-period spectrum.

perfectly reflective. The long-wave spectrum (Figure 2; Nishii et al., 2008) ranging from $0.003 \mathrm{~Hz}$ $(300 \mathrm{~s})$ to $0.05 \mathrm{~Hz}(20 \mathrm{~s})$, expressed with an exponential function taking a maximum value at a peak frequency, is used for the incident wave spectrum. The shape of the spectrum approximates a wave spectrum observed by NOWPHAS (Nationwide Ocean Wave Information Network for Ports and Harbors) at the nearby offshore island of Biro. The incident random waves, which consist of 512 component waves having the same energy and different phases produced by standard random numbers, are generated at the wave generating boundary. Setting the computational grid length $25 \mathrm{~m}$, and the time increment $0.25 \mathrm{~s}$, computations are performed.

\section{NUMERICAL CALCULATIONS}

\section{Thickness of the Sponge Layer}

In the case that only wind waves are considered, the thickness of the sponge layer is usually about twice the significant wave length to almost completely absorb the reflective waves from the land boundaries or breakwaters. In the case that long-waves are considered, the sponge thickness needs to be larger according to wavelength, and thus, the computational region becomes significantly larger. Nishii et al. (2007) investigated wave absorbing characteristics of the sponge layer, by performing numerical experiments using a one-dimensional numerical wave-tank model. The results show that the sponge thickness needs to be about twice the target wave length to almost perfectly absorb the reflective waves. Therefore, at a wave period of $300 \mathrm{~s}$, for example, the sponge thickness at the wave generating boundary (depth of $20 \mathrm{~m}$ ) needs to be no less than $8400 \mathrm{~m}$.

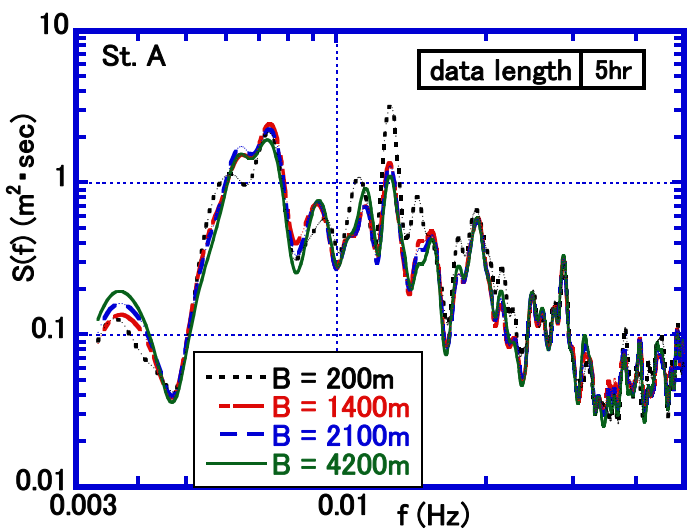

Figure 3(a). Spectral variation due to sponge thickness.

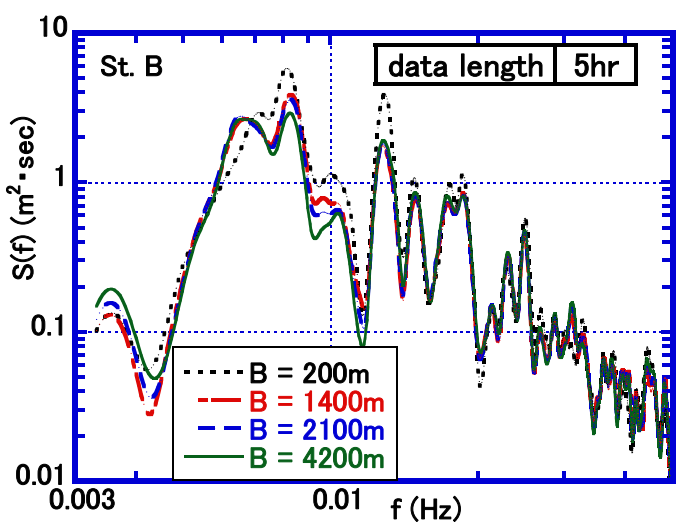

Figure 3(b). Spectral variation due to sponge thickness.

In the plane field calculations, however, each component wave is generally incident on the sponge layer at certain angles; the sufficient sponge thickness depends on the incident angle and wavelength. To clarify how greatly the sponge thickness affects the plane field computations in the case of irregular long-waves, computations for several sponge thicknesses (4200, 2100, 1400, and $200 \mathrm{~m}$ ) were made, and the spectra of the water surface oscillations obtained at two points in the harbor (St. A and St. B 
shown in Figure 1) were compared (see Figures 3(a) and 3(b)). A sponge thickness of $4200 \mathrm{~m}$ is equivalent to the wavelength of a wave with a period of $300 \mathrm{~s}$. This sponge thickness is sufficient to completely absorb component waves with a period $100 \mathrm{~s}$, but for component waves with a period of $200 \mathrm{~s}$, waves with $30 \%$ of the incident wave height are reflected back into the sea field according to the results of Nishii et al. Therefore, it was expected that the shorter the sponge thickness becomes, the more multi-wave-reflection in the sea field would occur, and consequently, the spectra obtained for different sponge thicknesses would vary significantly from each other. Contrary to what we expected, the influence of the sponge thickness is relatively small, as shown in Figures 3(a) and (b). In particular, the spectra in the period range from 30 to $120 \mathrm{~s}$, where the natural periods of relatively large ships lie, are almost the same, except for a sponge thickness of $200 \mathrm{~m}$.

Taking the spectrum for which the sponge thickness is the maximum, $4200 \mathrm{~m}$, as the standard spectrum, $\bar{S}(f)$, the deviation of each spectrum against the standard spectrum obtained at St. A is shown in Figure 4. It is found that the greater the sponge thickness becomes, the less deviation occurs, and the spectrum for the $3500 \mathrm{~m}$ sponge thickness is almost the same as the standard spectrum. Moreover, the deviations for different sponge thicknesses are nondimensionalized using the standard spectrum and their mean square values are computed. Figures 5(a) and (b) show the mean square values against the sponge thickness. The mean square values decrease exponentially as the sponge thickness increases. It is shown that the variation in spectrum with sponge thickness is much smaller than expected from the one-dimensional investigation conducted by Nishii et al. This is a very favorable result in terms of performing practical computations for a number of different conditions. The reason that the effect of the sponge thickness is much less than expected from one-dimensional numerical experiments is not certain at present. However, it can be pointed out that waves are generally incident on the sponge layer at different angles, and thus the ratio of the sponge thickness to wavelength is larger than in the case of normal incidence. In this study, the computation of wave spectra and their examination were carried out for two monitoring points in the harbor, St. A and St. B as shown in Figures 3(a) and 3(b) and Figures 5(a) and 5(b). However, significant differences between St. A and St. B were not found in the following investigations. Therefore, subsequent discussions refer only to St. A.

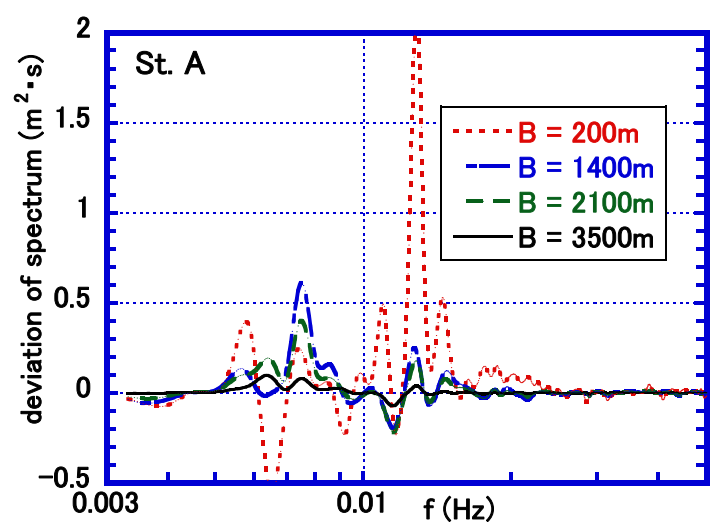

Figure 4. Deviations of spectra from the standard spectrum.

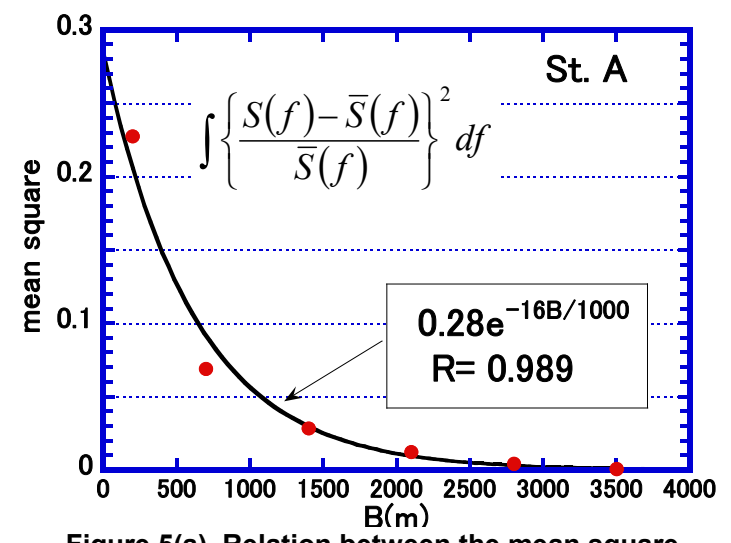

Figure 5(a). Relation between the mean square of the relative deviation and sponge thickness.

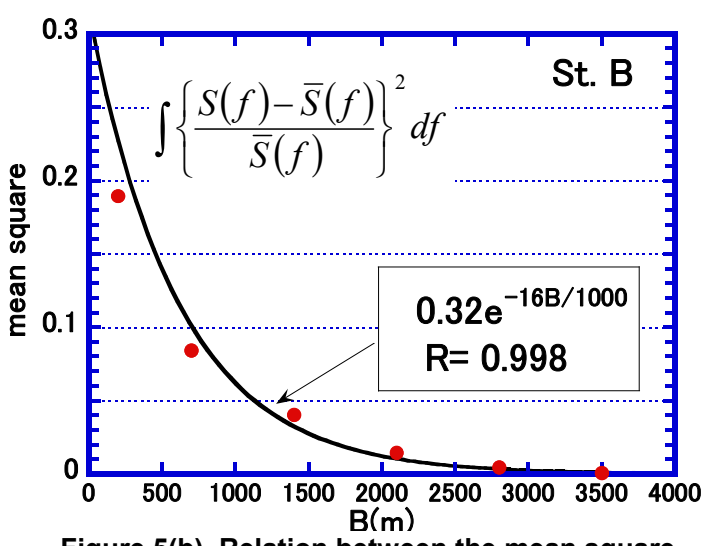

Figure 5(b). Relation between the mean square of the relative deviation and sponge thickness. 

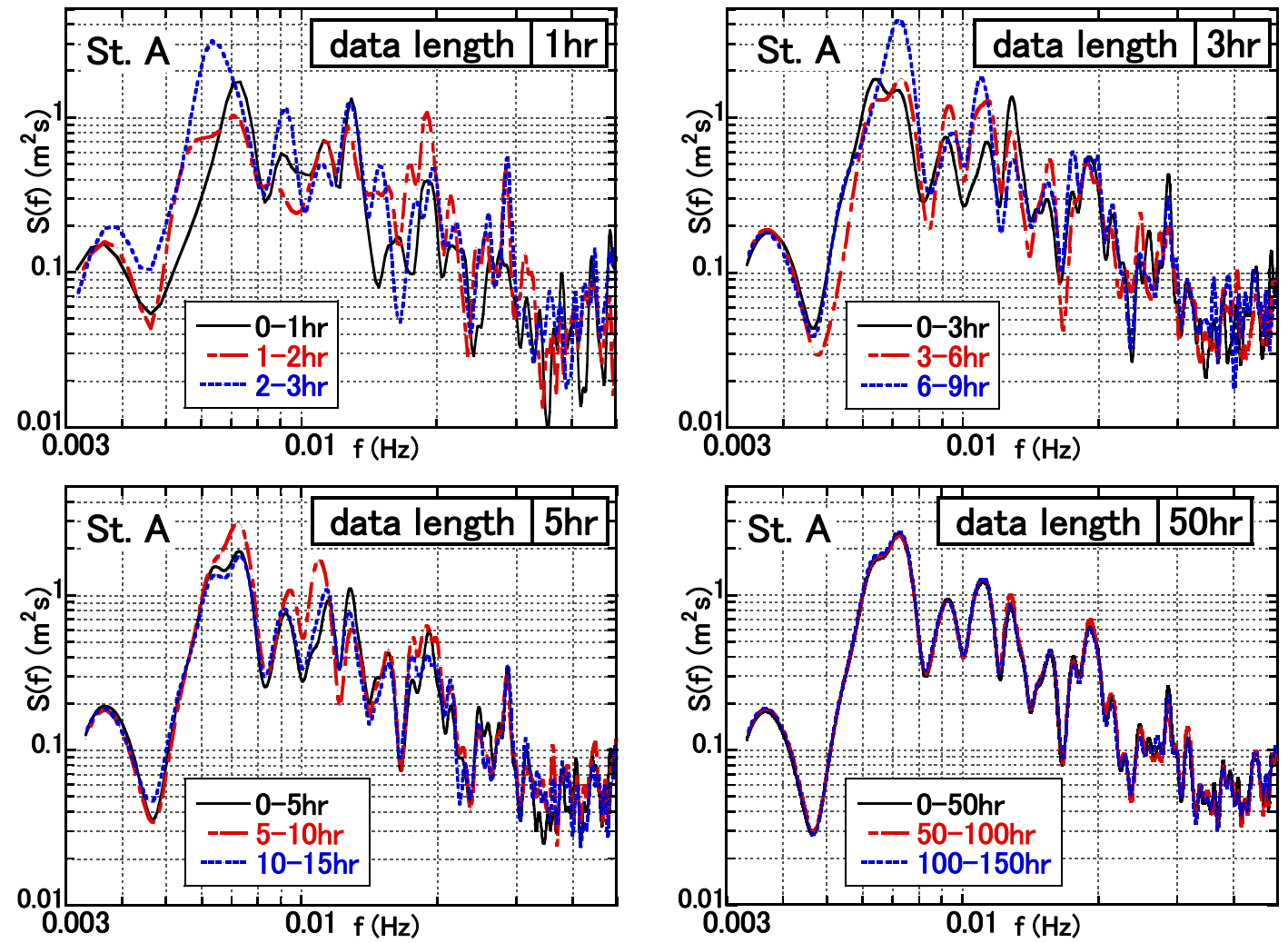

Figure 6. Spectral variation and convergence due to data length.

\section{Computing Time}

It is accepted that the computation time needs to be longer than the one in the case of wind waves to get statistically reliable spectra. Although 1 to $2 \mathrm{~h}$ of computing time seems to be widely used to estimate the long-wave field, how long the computation time should be in order to get an equilibrium spectrum of long period waves is not certain.

To investigate the effects of the computing time on the long-wave spectra in harbors, a very long computation $(200 \mathrm{~h})$ was performed. Spectra of the water surface oscillations at St. A were calculated for 13 data lengths ranging from $40 \mathrm{~min}$ to $50 \mathrm{~h}$. 200 spectra were obtained for a data length of $1 \mathrm{~h}$ and four spectra for a data length of $50 \mathrm{~h}$. Figure 6 shows three successive spectra obtained for 1, 3, 5, and $50 \mathrm{~h}$ data lengths. It is seen that spectra of a data length of $1 \mathrm{~h}$ differ markedly, and that spectral

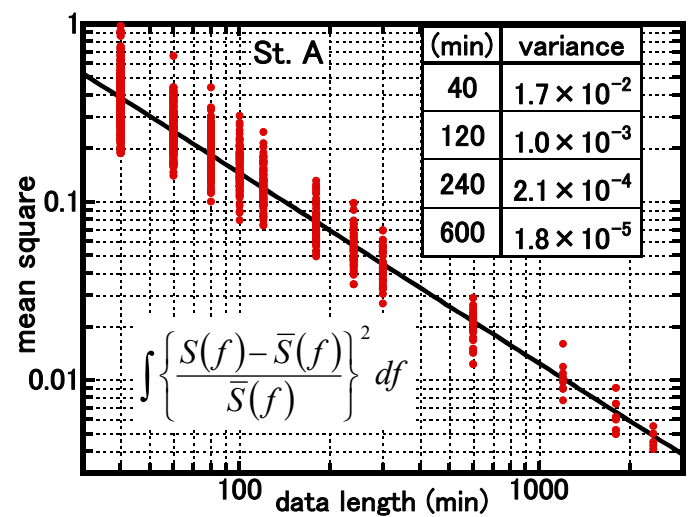

Figure 7. Relation between the mean square of the relative deviation and data length.

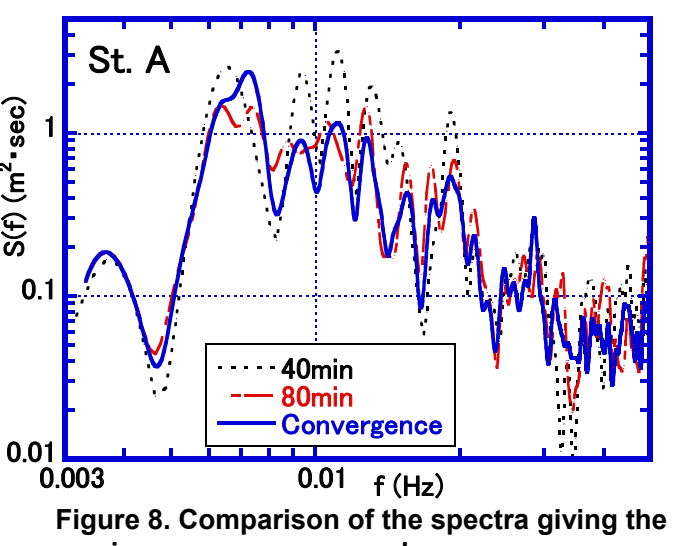

maximum mean square value. 
variations remain in some degree for a data length of $5 \mathrm{~h}$. On the other hand, spectra of a data length of $50 \mathrm{~h}$ seem to coincide almost completely. Figure 7 shows the mean square values for the relative deviation of spectra of different data lengths against the equilibrium spectrum (the spectrum of data length $50 \mathrm{~h}$ ). The line in the figure is the regression curve based on mean square values, and variances of mean square values for each data length are given in the figure. It is seen from the figure that the smaller the data length is, the greater the dispersion of spectra. Figure 8 shows a comparison of the equilibrium spectrum with spectra taking the maximum mean square values for data lengths of 40 and $80 \mathrm{~min}$. Even using a data length of $80 \mathrm{~min}$ can lead to great differences from the equilibrium spectrum.

To quantify spectral variations due to data length, long-period significant wave heights and periods were calculated by the zero-upcrossing method. Figure 9 shows the variation in the long-period significant wave height due to data length. It is seen that in case of a data length of $1 \mathrm{~h}$, difference between the maximum and the minimum is about $18 \mathrm{~cm}$, which is equivalent to $45 \%$ of the convergence value $40 \mathrm{~cm}$.

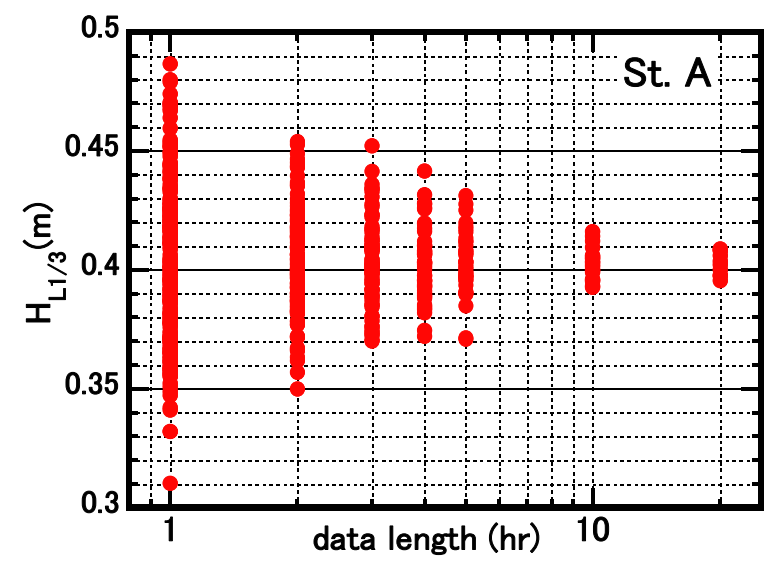

Figure 9. Variation in long-period significant wave height due to data length.

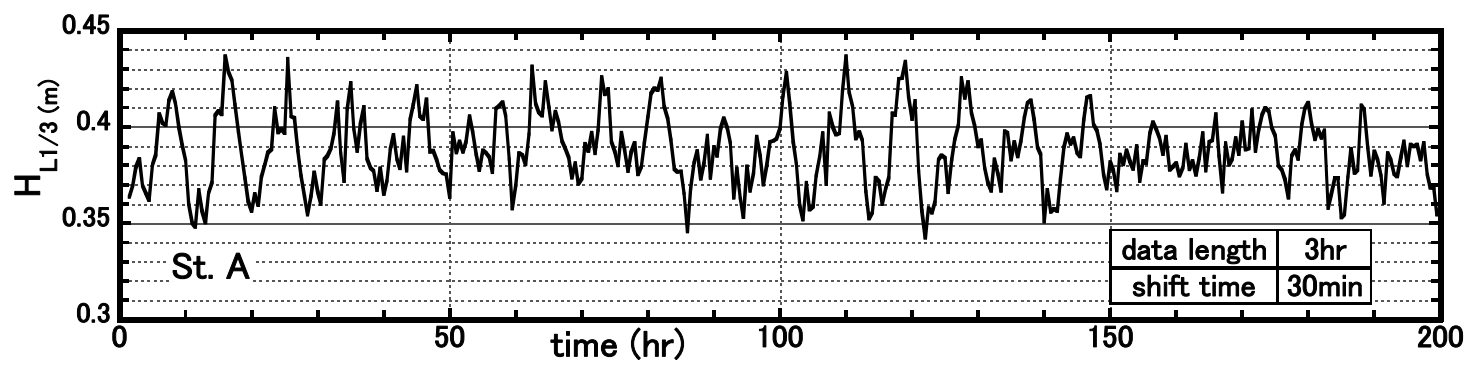

Figure 10(a). Time series of the long-period wave height.

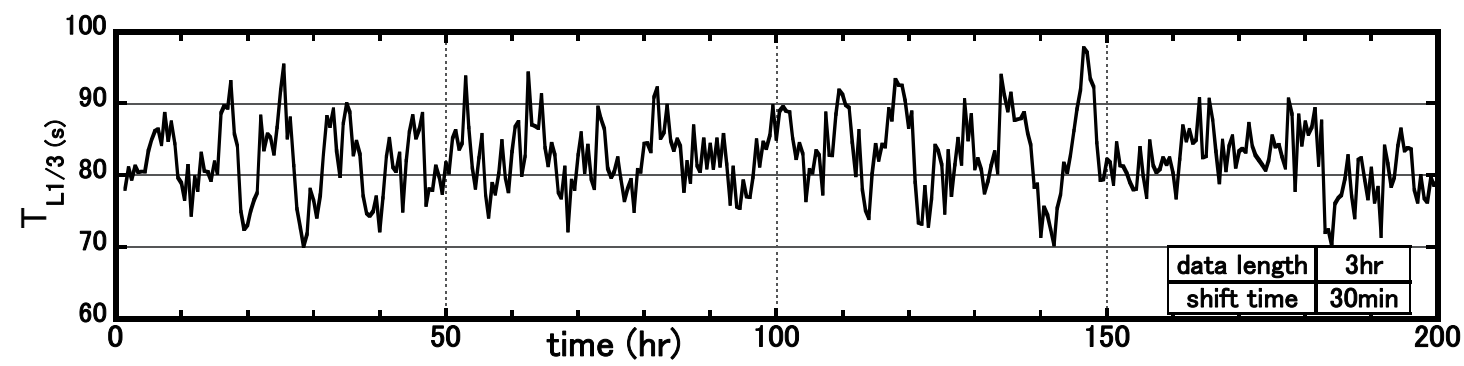

Figure 10(b). Time series of the long-period wave period. 
Looking at the equilibrium spectrum in Figure 6, it is clearly seen that there are four spectral peaks at frequencies ranging $0.007-0.014 \mathrm{~Hz}$. The data length of $4-5 \mathrm{~h}$ can include at least 100 waves of these frequency components and therefore significant values should almost converge by analogy with the statistical theory of wind waves. However, a glance at Figure 9 reveals that proper convergence does not seem to be achieved for a data length of $4-5 \mathrm{~h}$, and even if employing a data length of $10 \mathrm{~h}$, a difference of some centimeters remains. To find the reason for the variability seen in spectra of the 4-5 $\mathrm{h}$ data length, time series of long-period significant wave heights and periods were obtained by taking data lengths of 3 and $5 \mathrm{~h}$ and shifting the data range by $30 \mathrm{~min}$. Figure 10(a) and 10(b) shows the results of using a data length of $3 \mathrm{~h}$. The figures both show very distinct periodic oscillations and their periods are read around $9 \mathrm{~h}$, which is much longer than the time needed to gain statistically reliable spectra.

(a) (b) (c)

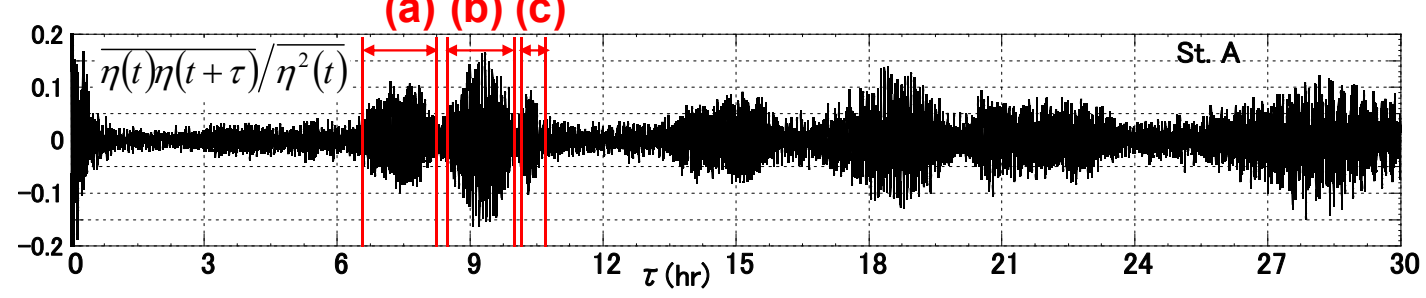

Figure 11. Autocorrelation of water surface oscillation.

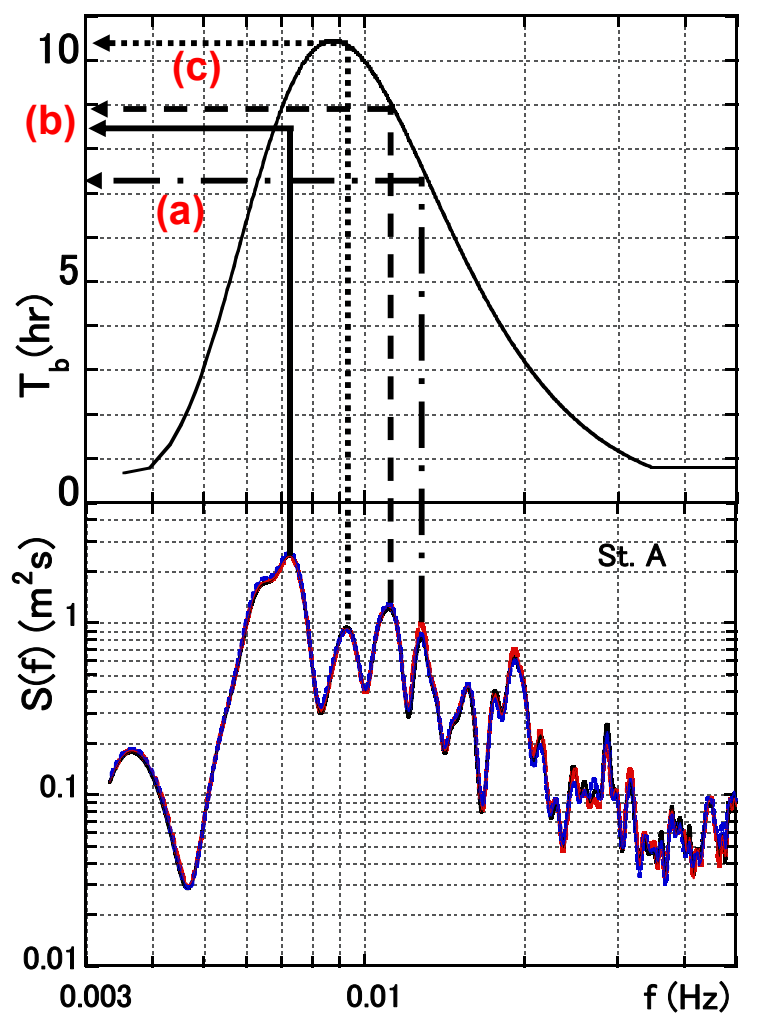

Figure 12. Relation between beat periods produced by adjacent frequency components and harbor resonance characteristics.

To clarify the periodicity shown in Figure 10(a) and (b), autocorrelation coefficients (Figure 11) of the water surface oscillation at St. A were calculated. Clear correlations appeared in three intervals of (a) 6.5-8.0, (b) 8.5-9.6, and (c) 9.6-10.0 h, and the repetitions continued until the end of the 
observation. The cause of these periodic variations in significant waves can be explained in the followings. Random incident waves are produced on a basis of the spectrum shown in Figure 2 so that each component wave has the same energy. Adjacent frequency components of the incident spectrum generally make beats. Figure 12 (top figure) shows the beat period distribution against the frequency component. Because the adjacent frequency components around the spectrum peak become especially close to each other, some of the beat periods reach over 10 hours. On the other hand, harbors generally have their own resonance characteristics, for example, as seen in Figure 12 (bottom figure), and they selectively amplify the incident waves at the resonance frequencies. It is seen from Figure 12 that several strong resonances appear at frequencies ranging 0.007-0.014 Hz and these resonance frequencies correspond to the period ranges, (a), (b), and (c), seen in the autocorrelation in Figure 11. It can be concluded that the resonance modes intensify the beats with longer beat periods, and therefore, the spectrum, which is usually obtained using less data length than the beat periods, varies in proportion to the data length. And this also explains the fluctuations in Figure 10(a) and (b).

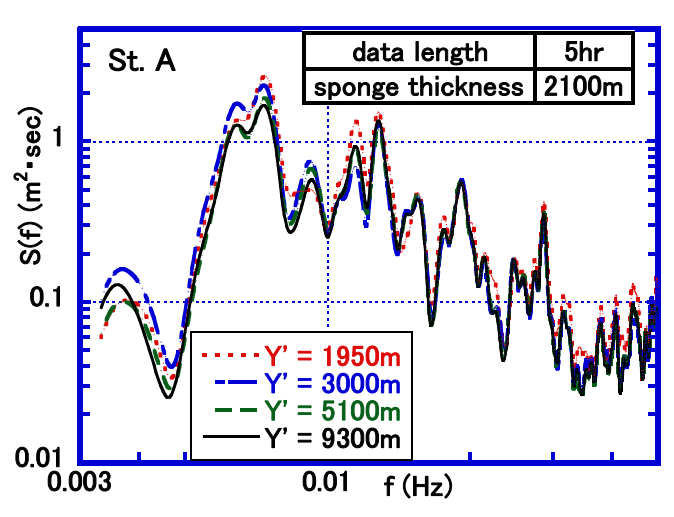

Figure 13. Spectral variation due to $Y^{\prime}$.

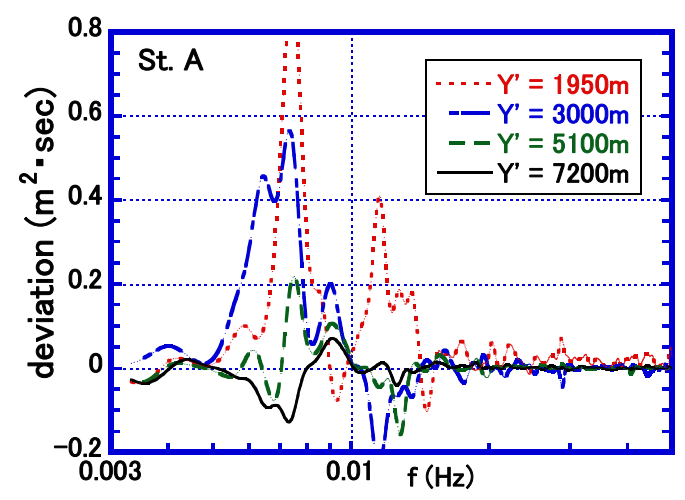

Figure 14. Deviations of spectra against the standard spectrum.

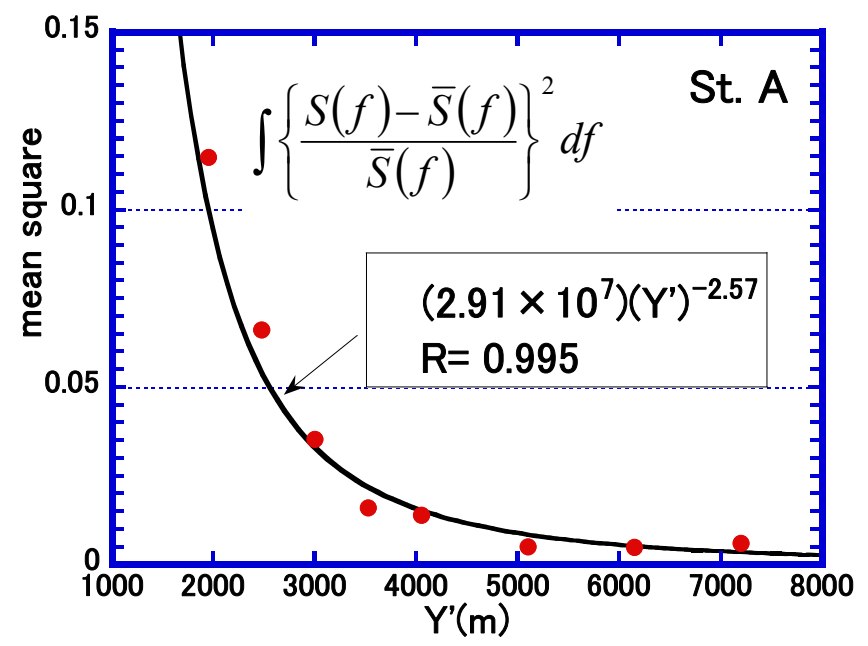

Figure 15. Relation between the mean square of the relative deviation and $Y^{\prime}$. 


\section{Size of the Sea Area}

The size of the computational area including a harbor seems often to be determined empirically. However, since the wave energy going into a harbor varies according to the distance Y' (see Figure 1) between the end of the breakwater and the lateral sponge boundary, it is clear that the size of the computational area has an influence on the water surface oscillations in the harbors.

To investigate the influences of the size of the computational area, computations were performed for a number of $\mathrm{Y}^{\prime}$ values from $1950 \mathrm{~m}$ to $9300 \mathrm{~m}$. The distance between the wave generation boundary and the coastline was kept constant at $7100 \mathrm{~m}$. Figure 13 compares spectra obtained for four values of Y'. Naturally, the lower the frequency is, the greater the difference appears. To clarify the differences among the spectra, the spectrum for which $\mathrm{Y}^{\prime}$ is a maximum of $9300 \mathrm{~m}$ is defined as the standard spectrum, and the deviation of each spectrum against the standard spectrum and the mean square of the relative deviation were obtained as shown in Figures 14 and 15 respectively. From Figures 13,14 , and 15, it is seen that the size of the computational area does not have a great effect where the wave period is less than about $60 \mathrm{~s}$. On the other hand, reducing the size of the computational area greatly influences the spectra where the period is greater than $60 \mathrm{~s}$. The variation in the spectrum with the size of the computational area appears only at peak frequencies.

\section{CONCLUSIONS}

The sponge thickness, computing time, and the size of the computational area when calculating the long-wave field using the Boussinesq model were discussed. Significant results obtained in this study are summarized as follows:

1. According to the one-dimensional numerical wave-tank experiments (Nishii et al.), the sponge thickness for sufficiently absorbing the reflective waves needs to be about 2 to 3 times the incident wave length. However, in plane wave field calculations, it was found that the sponge thickness influences the spectrum less than is predicted, and that stable computations can be obtained without causing multiple reflections, even if the sponge thickness is much less than the ideal thickness recommended from one-dimensional computations.

2. Although a computing time of 1 to $2 \mathrm{~h}$ has been widely used without any explicit basis, it is too short to obtain a stationary spectrum and a much longer time is needed; e.g., from statistical point of view, 3-5 h is needed to include 100-200 waves if the target wave period is $100 \mathrm{~s}$.

3. However, even if a data length of $3-5 \mathrm{~h}$ is used, the spectra vary according to where the data is extracted from the time series of water surface oscillations, and much longer data length is generally required to obtain converged spectra. This can be explained that frequency component waves in the incident wave generally make beats, and harbor resonance selectively amplifies component waves near resonant frequencies, and consequently, the beats produced by the amplified adjacent component waves greatly affect the time series of water surface oscillations.

4. Spectral shapes vary according to the size of the computational area, and the lower the frequency is, the more variation appears. The variation in the spectrum appears only at peak frequencies. The distance $3 \mathrm{~km}$ between the end of the breakwater and the lateral sponge boundary is practically sufficient.

5. Comparing the mean square values of relative deviations against ideal conditions for sponge thickness, computing time, and size of the computational area (Figures 5, 9, and 13), it is seen that the computing time makes the greatest difference by far among the three conditions. In other words, unless the computing time is sufficiently long, it is not worth discussing the influences of the sponge thickness and the size of computational area.

The future direction of this study will be the detailed investigation into the Boussinesq model for long-wave fields through comparison of field measurements and computations. This study contributes basic information about computational conditions that will be used for that work.

\section{ACKNOWLEDGMENTS}

Miharu Ishikawa (Yoshii System Research Inc.) is acknowledged for improving the Boussinesq model program and for other helpful support. Computations were mainly carried out using the computer facilities at the Research Institute for Information Technology, Kyushu University. This work was supported by the International Training Program of the Japan Society for the Promotion of Science. 


\section{REFERENCES}

Bingham, H.B. 2000. A hybrid Boussinesq-panel method for predicting the motion of a moored ship, Coastal Engineering, 40, 21-38.

Fujihata, S., Hata, S., Moriya, Y., Nakayama, S., Sekimoto, T. 1998. Field Observation on Properties of Long Period Wave Velocity in a Harbor and Its Numerical Simulation, Proceeding of Coastal Engineering, JSCE, Vol.45, pp.306-310. (in Japanese)

Hiraishi, T., Hirayama, K. 2003. Applicability of Wave Transformation Model in Port, Proceedings of Civil Engineering in the Ocean, Vol.19, pp.65-70. (in Japanese)

Hirayama, K. 2002. Utilization of Numerical Simulation on Nonlinear Irregular Wave for Port and Harbor Design, Technical Note of the Port and Airport Research Institute, No.1036, pp.69-89. (in Japanese)

Madsen, P.A., R. Murray and O.R. Srensen. 1991. A new form of the Boussinesq equations with improved linear dispersion characteristics, Coastal Engineering, 15, pp.371-388.

Nishii, Y., Yoshida, A., Yamashiro, M. 2007. Basic Verification on Boundary Procedures in Boussinesq Calculation for Long-period Waves, Proceedings of Civil Engineering in the Ocean, Vol.23, pp.153-158. (in Japanese)

Nishii, Y., Yoshida, A., Yamashiro, M., Ono, T. 2008. Proposition of long Period Wave Spectra for Numerical Estimation of Wave Field in Harbors and Its Verification with Field Data, Proceeding of Coastal Engineering, JSCE, Vol.54, pp.256-260. (in Japanese)

Wehausen, J.V. 1971. The Motion of Floating Bodies, Annual Review of Fluid Mechanics, Vol.3, 237268.

Yoshida, A., Nishii, Y., Yamashiro, M. and Ohta, K. 2008. Estimation of wave exciting forces on moored ships in harbors through wave field calculations with Boussinesq model, Proceedings of $31^{\text {th }}$ International Conference on Coastal Engineering, ASCE, 735-747. 